\title{
Supervivencia y crecimiento de plantines de ciprés de la cordillera durante siete años en dos sitios contrastantes de Patagonia norte
}

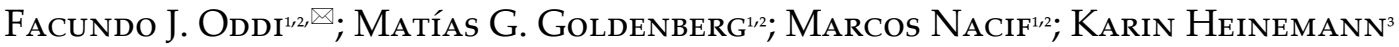 \\ \& LuCAS A. Garibaldi ${ }^{1 / 2}$
}

${ }^{1}$ Universidad Nacional de Río Negro. Instituto de Investigaciones en Recursos Naturales, Agroecología y Desarrollo Rural. Río Negro, Argentina. ${ }^{2}$ Consejo Nacional de investigaciones Científicas y Técnicas. Instituto de Investigaciones en Recursos Naturales, Agroecología y Desarrollo Rural. Río Negro, Argentina. ${ }^{3}$ Sociedad Naturalista Andino Patagónica (SNAP).

Bariloche, Río Negro, Argentina.

\begin{abstract}
RESUMEN. Las especies forestales nativas permiten restaurar bosques y son una alternativa productiva sustentable. Como en cualquier forestación, en bosques implantados o enriquecidos con nativas, el conocimiento sobre la dinámica de los plantines resulta clave para establecer estrategias de manejo. En este estudio describimos la dinámica de la supervivencia y el crecimiento en altura en plantines de ciprés de la cordillera (especie nativa de los bosques Andino-patagónicos de alto valor forestal) en matorrales de la Patagonia norte. Establecimos un experimento de plantación ( 275 plantines) en dos sitios contrastantes (un fondo de valle y una ladera de exposición norte) y durante 6 ó 7 años, dependiendo del sitio, en cada primavera registramos la supervivencia y la altura de los plantines. Evaluamos además el efecto de la protección contra herbívoros y modelamos la dinámica del ramoneo. La supervivencia y el ramoneo la evaluamos con modelos lineales generalizados, y para la altura ajustamos un modelo no lineal de efectos mixtos que permitió cuantificar la tasa de crecimiento de cada plantín. En el fondo de valle, la supervivencia disminuyó hasta menos del $15 \%$ al finalizar el experimento. En la ladera de exposición norte, la supervivencia fue más alta y se mantuvo relativamente estable. En este sitio, los valores de supervivencia ( $70 \%$ al séptimo año) y crecimiento en altura (tasas mayores al $15 \%$; IMA $\sim 8 \mathrm{~cm} /$ año) fueron comparables a los reportados en plantaciones bajo bosque de ciprés de la cordillera, lo que sugiere que la especie se podría utilizar para restaurar/producir en matorrales mixtos de exposición norte. Los protectores redujeron el ramoneo, aumentaron la supervivencia, aunque no significativamente, y atenuaron el crecimiento. Por lo tanto, no es clara la conveniencia de proteger los plantines con el tipo de protector evaluado. Esperamos que nuestros resultados contribuyan con la gestión sustentable de los matorrales y bosques de la Patagonia norte.
\end{abstract}

[Palabras clave: Austrocedrus chilensis, plantación forestal con especies nativas, herbivoría, modelo no lineal de efectos mixtos]

\begin{abstract}
Авsтract. Survival and growth of 'ciprés de la cordillera' seedlings over seven years at two contrasting sites in northwestern Patagonia. Native forestry species enable forest restoration and are a sustainable productive alternative. In any forest plantation - among them those in which native tree species are used - knowledge on seedling dynamics is a highly relevant for planning and management. We describe the seedling dynamics - survival and height growth - of planted 'ciprés de la cordillera', a high-value timber species of the Andean-Patagonian forests, in northern Patagonia shrublands. We carried out a 7-years planting experiment (275 seedlings) in two contrasting sites (a valley bottom and a northern-exposure hillside), and each spring we recorded the survival and height of the seedlings. In addition, we evaluated the effect of protecting the seedlings from herbivores and modeled the browsing dynamics. Survival and browsing were evaluated with generalized linear models while height was modeled with a non-linear mixed-effects model from which we quantified the growth rate per seedling. At the valley bottom, survival decreased to $<15 \%$ at the end of the experiment. Survival was higher in the hillside with north exposure and remained relatively stable. At this site, the values of survival ( $70 \%$ at year 7 ) and height growth (rates greater than $15 \%$; MAI $\sim 8 \mathrm{~cm} /$ year) were comparable with those of seedlings planted in forests of 'ciprés de la cordillera', suggesting that the species could be used for restoration or production in northern hillsides like the one assayed. Seedling protectors reduced browsing, increased survival although not significantly, and diminished growth. Therefore, it is not clear whether seedlings should be protected using the protector type tested in this study. We hope our results contribute to the sustainable management of shrublands and forests of northern Patagonia.
\end{abstract}

[Keywords: Austrocedrus chilensis, native species forestry, herbivory, non-lineal mixed-effects model]

Editora asociada: María Semmartin Editora junior: Mariana Tadey

\footnotetext{
foddi@unrn.edu.ar
}

Recibido: 3 de Septiembre de 2020

Aceptado: 17 de Diciembre de 2020 


\section{INTRODUCCIÓN}

Los bosques nativos son ecosistemas claves para el bienestar humano (FAO 2018). Su gestión demanda que los objetivos productivos y económicos deban ser alcanzados bajo un marco de sustentabilidad ambiental (SteffanDewenter et al. 2007). Esto implica considerar al bosque como proveedor de múltiples bienes y servicios (Puetmann et al. 2011), y plantar especies nativas es una interesante alterativa de restauración/enriquecimiento de ecosistemas y producción forestal sustentable (Montagnini et al. 2004; Promis 2020). En Sudamérica, donde existen tasas de deforestación elevadas (FAO 2018), la información sobre manejo forestal se centra, paradójicamente, en plantaciones con especies exóticas de rápido crecimiento (Grosfeld et al. 2019).

El manejo forestal tiene sus bases en el conocimiento y comprensión de los cambios temporales en las variables estructurales del bosque (e.g., composición de especies, densidad, tamaño de los individuos, etc.), es decir, en su dinámica (Oliver and Larson 1996). La dinámica es particularmente importante durante los estadios iniciales, ya que corresponde al período en el cual las plantas son más vulnerables (PérezRamos and Marañón 2001). En bosques implantados, dos de los indicadores ecológicos de mayor relevancia son la supervivencia y el crecimiento de los plantines (Gizachew et al. 2012); la primera determina la cantidad de individuos en un momento dado y la segunda el tamaño de los mismos. Sus dinámicas, por lo tanto, se vinculan con las relaciones de tamaño-densidad que permiten, por ejemplo, planificar el momento óptimo de las intervenciones (Zeide 2004).

En la Patagonia, los matorrales, tanto mixtos como puros de ñire (Nothofagus antarctica [ñirantales]), constituyen uno de los tipos forestales más representativos. Se distribuyen entre los $37^{\circ}$ y los $56^{\circ}$ de latitud sur, abarcando un amplio gradiente altitudinal (Reque et al. 2007; Peri et al. 2013). Como consecuencia, estas comunidades son fisionómicamente variables, observándose desde coberturas arbustivas con individuos achaparrados hasta zonas donde los individuos desarrollan porte arbóreo (Ramírez et al. 1985). Mientras que al sur de la región forman una matriz pura de ñire de características boscosas, en latitudes menores a los $42^{\circ}$ se caracterizan por crecimientos más arbustivos y en parches de tamaño variable (Pastorino et al. 2009). En la Patagonia norte, en particular, estos ecosistemas han sido históricamente considerados de bajo valor económico y ambiental (Reque et al. 2007), y las plantaciones de coníferas exóticas y el uso silvopastoril representan las principales alternativas productivas. Esto implicó extracción o reemplazo de especies nativas, muchas veces con un consecuente proceso de degradación de la tierra que requiere de prácticas activas para su restauración (Rusch et al. 2017). Sin embargo, los matorrales son uno de los tipos forestales más diversos de los bosques Andino-patagónicos (Veblen et al. 1996), y su uso y conservación deben ser objeto de atención, ya que proveen múltiples servicios ambientales (Goldenberg et al. 2020b). Descontando la superficie dentro de áreas protegidas, en la Patagonia norte, los matorrales ocupan $\sim 60000$ ha, de las cuales un tercio son matorrales mixtos (Fundación INVAP y CIEFAP 2019). Actualmente, la ordenación territorial de la región (CIEFAP y MADyS 2016) estableció que una gran parte de toda esta área sólo se puede aprovechar bajo manejo sustentable (Ley Nacional 26331). Dado que los matorrales son ambientes adecuados para muchas especies nativas de interés forestal (Urretavizcaya et al. 2015), plantar estas especies podría servir para restaurar o enriquecer sitios degradados, a la vez que brindaría una alternativa de producción sustentable.

El ciprés de la cordillera (Austrocedrus chilensis (D. Don) Pic. Serm. et Bizzarri) es una de las especies forestales nativas que podría utilizarse para fines de restauración, enriquecimiento o producción. En ausencia de disturbios, tiende a dominar los matorrales mixtos en etapas sucesionales avanzadas (Kitzberger and Veblen 1999). Este proceso podría facilitarse mediante plantación, estableciendo un rodal coetáneo del que se podrían obtener productos de alta calidad maderable con un manejo relativamente simple. Por otro lado, en el corto y mediano plazo sería posible obtener productos dendroenergéticos (i.e., leña, chips) de las especies arbustivas rebrotantes (Goldenberg et al. 2020a) sin comprometer la biodiversidad del sitio (Goldenberg et al. 2020b). Además, los matorrales mixtos tienen numerosas especies que se podrían utilizar como nodrizas (Núñez et al. 2009), condición que beneficia la supervivencia y el desarrollo de los renovales de ciprés durante los primeros años (Kitzberger et al. 2000). En términos económicos, recientemente se demostró que podría ser una alternativa más rentable que una forestación de Pinus ponderosa (Dougl.) 
ex Laws (Goldenberg et al. 2018), la conífera exótica más plantada en la Patagonia.

El amplio conocimiento sobre la ecología de los bosques de ciprés(Veblen etal.1995; Donoso Zegers 2013) y su dinámica de regeneración (Kitzberger et al. 2000; Rovere 2004; Núñez et al. 2009) contrasta con el obtenido en experimentos de plantación (Pafundi et al. 2014). Son pocos los ensayos de plantación de ciprés, y de especies nativas en general, que cuentan con parcelas permanentes y realizan un seguimiento temporal de las respuestas ecológicas (De Paz et al. 2019). La mortalidad del ciprés puede ser significativa al primer o segundo año post-plantación (Urretavizcaya et al. 2015 y referencias allí contenidas), pero se desconoce el momento en el que la tasa de supervivencia se estabiliza; esta variable se suele monitorear durante 3 años como máximo (De Paz et al. 2019). En relación con el crecimiento, en general, los modelos han sido ajustados en bosque nativo y a partir de la información retrospectiva que brindan los anillos de crecimiento (Goya et al. 1998; Navarrete Espinoza et al. 2008; Landesmann et al. 2016). Los estudios que incluyeron series temporales de altura son pocos y de corta duración; estos trabajos sugieren avanzar hacia el uso de modelos que permitan cuantificar la tasa de crecimiento en forma directa (Aparicio et al. 2009; Aparicio et al. 2010). En este sentido, los modelos no lineales de efectos mixtos presentan interesantes ventajas para describir dinámicas temporales de variables ecológicas (Oddi et al. 2019). Por otro lado, entre los factores decisivos para la supervivencia y crecimiento en plantaciones forestales con especies nativas se encuentra la herbivoría (De Paz et al. 2019). Es común que los renovales de ciprés sean ramoneados (Relva and Sancholuz 2000), lo cual puede reducir el crecimiento y deformar el fuste (Relva and Veblen 1998), con consecuencias también sobre la supervivencia (Rovere et al. 2004). La liebre europea (Lepus europaeus Pallas 1778), en particular, es uno de los herbívoros más problemáticos (Gowda et al. 2014), al punto que se lo considera plaga forestal en la región (Bonino 2009). Si bien para su control se suelen colocar clausuras o protectores individuales, esta es una difícil decisión para el productor, ya que esta práctica es costosa y técnicamente compleja (Rusch et al. 2017).

La factibilidad y la viabilidad de las plantaciones de especies nativas es poco conocida en los matorrales de la Patagonia norte (Urretavizcaya et al. 2015), lo cual limita el uso del ciprés con fines de restauración, enriquecimiento o producción. El objetivo de este estudio fue modelar la dinámica de la supervivencia y el crecimiento inicial en altura de dos plantaciones de ciprés. Abordamos este objetivo mediante un experimento de campo de 7 años, evaluando ambas respuestas en dos sitios de matorral contrastantes considerando además el efecto de la protección contra la liebre.

\section{Materiales y Métodos}

\section{Área de estudio y sitios de plantación}

Establecimos parcelas permanentes sobre dos sitios ambientalmente contrastantes.

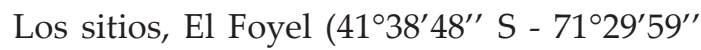

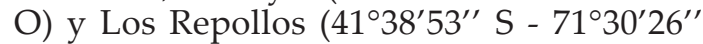
O), distanciados $30 \mathrm{~km}$ entre sí, se localizan entre Bariloche y El Bolsón (Provincia de Río Negro, Argentina). El Foyel es una ladera de exposición norte y Los Repollos un fondo de valle. La altitud de ambos sitios es similar (790 m s. n. m. en El Foyel y 880 m s. n. m. en Los Repollos), y se caracterizan por un clima templado-frío con precipitaciones anuales entre 700 mm (Los Repollos) y 900 mm (El Foyel), concentradas durante otoño-invierno. Las temperaturas medias anuales de estos sitios varían entre $8{ }^{\circ} \mathrm{C}$ (El Foyel) y $9{ }^{\circ} \mathrm{C}$ (Los Repollos), con máximas promedio de 15 ${ }^{\circ} \mathrm{C}$ y mínimas de $1.5^{\circ} \mathrm{C}$. La influencia de la topografía genera que en El Foyel, el período de heladas dure mucho menos que en Los Repollos; la cantidad de días con heladas en verano es la mitad (Tabla A1 del Material Suplementario) y, presumiblemente, su intensidad también sea menor. El Foyel tiene suelos del grupo de los Hapludoles, oscuros, poco arenosos y con abundante densidad de raíces. En Los Repollos, los suelos son más someros y pertenecen al grupo de los Udivitrantes, menos oscuros, arenosos con bloques finos y de menor densidad radical (más información es provista en la Tabla A1 del MaterialSuplementario). Fitogeográficamente, la localización de los sitios pertenece al dominio Sub-antártico. Estos matorrales pueden estar dominados por formas arbóreas o arbustivas de Nothofagus antarctica (G. Forst.) Oerst.) (ñire) o co-dominado por otras especies leñosas, y constituyen uno de los tipos forestales más representativos de los bosques caducifolios de la Patagonia Andina (Veblen et al. 1996). Su estructura forestal actual responde al intensivo uso antrópico que históricamente se le ha dado a estas comunidades: pastoreo y extracción de leña (Gyenge et al. 2009). En el Foyel ocurre 
un matorral mixto y relativamente abierto. Las especies leñosas más representativas de este sitio son N. antarctica, Diostea juncea (Gillies ex Hook.) Miers, Schinus patagonica (Phil.) I. M. Johnst. ex Cabrera, Discaria chacaye (G. Don) Tortosa, Fabiana imbricata Ruiz et Pavón y Lomatia hirsuta (Lam.) Diels ex J.F. Macbr. (Gyenge et al. 2009). Estructuralmente, presenta una altura dominante de $4.4 \mathrm{~m}$, un área basal de $46.7 \mathrm{~m}^{2} / \mathrm{ha}$ y un diámetro cuadrático medio de $7.3 \mathrm{~cm}$. En Los Repollos se encuentra un matorral puro de $N$. antarctica, con una altura dominante de $2.1 \mathrm{~m}$, un área basal de $23.5 \mathrm{~m}^{2} /$ ha y un diámetro cuadrático medio de $2.8 \mathrm{~cm}$.

\section{Experimento de plantación}

Durante el otoño-invierno de 2008 plantamos 125 plantines de ciprés en El Foyel y 150 en Los Repollos, identificando a cada uno de ellos para su posterior seguimiento hasta el año 2014 en Los Repollos y hasta 2015 en el Foyel. Los plantines fueron plantados del lado sur de un arbusto. Usamos hileras de plantación separadas en aproximadamente $4 \mathrm{~m}$ y con una distancia entre plantines de 3 m (siempre dependiendo de la configuración espacial de los arbustos), lo que representa $\sim 833$ plantas/ha. Esto resultó en una superficie de plantación de aproximadamente un cuarto de hectárea en cada sitio. En cada hilera, a la mitad los plantines les aplicamos un protector individual para evitar el daño por el ramoneo de la liebre (malla metálica cilíndrica de rezago de chapa corona, $30 \mathrm{~cm}$ de diámetro y $50 \mathrm{~cm}$ de alto). Luego de la plantación, en cada primavera (excepto la de 2011 debido a la erupción del complejo volcánico Puyehue - Cordón Caulle) registramos supervivencia, presencia de ramoneo y altura de todos los plantines (6 años de datos en Los Repollos y 7 en El Foyel).

Los plantines utilizados provinieron del mismo vivero. Estos fueron repicados de almácigo a maceta (sachet de $750 \mathrm{~cm}^{3}$ ) un año antes del estudio. Usamos plantines de tres y cuatro años ya que experiencias previas mostraron que los plantines de menor edad presentan una mortalidad elevada (WilliamsLinera et al. 2011).

\section{Análisis de datos}

La supervivencia es una variable dicotómica cuya dinámica, a priori, podría modelarse mediante una estructura de datos binomial. Sin embargo, observamos que la dispersión fue mayor a la esperada bajo esta distribución. Por eso utilizamos un modelo lineal generalizado con distribución cuasi-binomial que incorpora un parámetro de sobre-dispersión. Los predictores del modelo fueron el tiempo desde la plantación (continuo) y su interacción con el sitio (categórico de dos niveles) y con la protección (categórico de dos niveles). La edad del plantín (categórico de dos niveles) también fue incluida como covariable:

$$
v_{i} \sim \text { cuasi binomial }\left(s_{i} ; n_{i}^{\prime} ; \phi\right)_{\text {indevendientes }}
$$

Ecuación 1

$$
\begin{aligned}
& \operatorname{logit}\left(s_{i}\right)=\beta_{0}+\beta_{1} T_{i}+\beta_{2} S_{i}+\beta_{3} P_{i}+ \\
& \beta_{4} E_{i}+\beta_{5}(T * S)_{i}+\beta_{6}(T * P)_{i}
\end{aligned}
$$

\section{donde}

$v_{i}=$ número de plantines vivos al tiempo $i$ (respuesta cuantitativa discreta),

$s_{i}=$ probabilidad de que un plantín esté vivo al tiempo $i$ (indica la tasa de supervivencia),

$$
\begin{aligned}
& n_{i}^{\prime}=\text { número de plantines al tiempo } i . \\
& \phi=\text { parámetro de sobre-dispersión, } \\
& T=\text { tiempo (en años) transcurrido desde la } \\
& \text { plantación (predictor continuo), }
\end{aligned}
$$

$S=$ sitio de plantación (predictor dicotómico: El Foyel, $S=0$; Los Repollos, $S=1$ ),

$E=$ edad del plantín al plantarse (predictor dicotómico: 3 años, $E=0 ; 4$ años, $E=1$ ),

$P=$ protección del plantín (predictor dicotómico: sin protección, $P=0$; protegido, $P=1)$.

En el caso del crecimiento en altura, lo evaluamos en los plantines que permanecían vivos al final del experimento (2015, es decir al séptimo año). Debido a la alta tasa de mortalidad que registramos en el sitio Los Repollos (fondo de valle), el modelo sólo incluyó los plantines del sitio El Foyel (ladera). La variable respuesta que modelamos fue la altura del plantín, y para esto ajustamos un modelo no lineal de efectos mixtos con distribución normal, modelando la heterocedasticidad en función del tiempo (varConstPower()), y la correlación temporal de los datos mediante una estructura auto-regresiva de medias móviles (ARMA) (Pinheiro and Bates 2000). Acorde a la estructura jerárquica impuesta 
por el diseño del experimento, el tiempo $(T)$ fue un predictor a nivel de observación (intraplantín), y la protección y la edad a nivel del plantín. El modelo asumió un crecimiento inicial de tipo exponencial $\left(y=a e^{b x}\right)$, con la tasa de crecimiento $(b)$ como una función de la protección $(P)$ y la edad del plantín $(E)$. En el caso de la ordenada al origen (a) solo permitimos que varíe en función de la edad; lógicamente, no consideramos el efecto de la protección al tiempo cero:

$h_{j i} \sim N\left(\mu_{i} ; \sigma_{i}^{2}\right)_{A R M A}$

$\sigma_{\mathrm{i}}^{2}=f\left(T_{\mathrm{i}}\right)$

$\mu_{i}=a_{j} * e^{b} T_{t}$

$a_{j} \sim N\left(\mu_{a_{j}} ; \sigma_{a}^{2}\right)$

$\mu_{a_{l}}=\alpha_{0}+\alpha_{1} E_{i}$

$b_{j} \sim N\left(\mu_{b_{j}} ; \sigma_{b}^{2}\right)$

$\mu_{b_{l}}=\beta_{0}+\beta_{1} P_{i}+\beta_{2} E_{i}$

donde

$h_{j i}=$ altura $(\mathrm{cm})$ del plantín $j(j=1,2, \ldots, 125$ menos número de muertos en el sitio sobre ladera) al tiempo $i$ (respuesta cuantitativa continua),

$a_{j}=$ altura del plantín $(\mathrm{cm}) j$ al momento de la plantación (esto es, a $T=0$ ),

$b_{j}=$ tasa de crecimiento en altura del plantín j,

$\sigma_{i}^{2}=$ varianza residual $\left(\mathrm{cm}^{2}\right)$ (variabilidad intra-plantín),

$\sigma_{a}^{2}=$ varianza de la altura al momento de la plantación $\left(\mathrm{cm}^{2}\right)$ (variabilidad entre plantines),

$\sigma_{b}^{2}=$ varianza de la tasa de crecimiento (variabilidad entre plantines).

Complementariamente, modelamos la dinámica del ramoneo. El ramoneo fue registrado como una variable dicotómica (presencia/ausencia) y por lo tanto ajustamos un modelo lineal generalizado de errores binomiales. Planteamos la misma componente determinística que en el caso de la supervivencia, es decir, el tiempo desde la plantación $(T)$ interactuando con el sitio $(S)$ y la protección $(P)$ y la edad del plantín (E) como covariable, pero incluyendo un término cuadrático en el tiempo $\left(T^{2}\right)$ para contemplar una posible respuesta no lineal. La no linealidad es esperable ya que al crecer y superar determinado tamaño, las plantas quedan fuera del alcance del animal modificando la tendencia temporal del ramoneo (si este umbral ocurre durante el período de análisis). El modelo que ajustamos fue el siguiente:

$$
r_{\mathrm{i}} \sim \text { binomial }\left(p_{i} ; n_{i}^{\prime}\right)_{\text {independientes }}
$$

Ecuación 3

logit $\left(p_{i}\right)=\beta_{0}+\beta_{1} T_{i}+\beta_{2} S_{i}+\beta_{3} P_{i}+$

$\beta_{4} E_{i}+\beta_{5}(T * S)_{i}+\beta_{6}(T * P)_{i}+\beta_{7} T_{i}^{2}$

donde

$r_{i}=$ número de plantines ramoneados al tiempo $i$ (respuesta cuantitativa discreta),

$p_{i}=$ probabilidad de que un plantín sea ramoneado al tiempo $i$ (indica la tasa de ramoneo),

$n_{i}^{\prime}=$ número de plantines al tiempo $i$.

Para los análisis usamos R 3.5.0 (R Core Team 2018). Los modelos de supervivencia (ecuación 1) y ramoneo (ecuación 3) los ajustamos con la función glm() del paquete Base y el modelo de crecimiento en altura (ecuación 2) con la función nlme() del paquete nlme (Pinheiro et al. 2016). Utilizamos un nivel de significancia de 0.05 .

\section{Resultados}

\section{Supervivencia}

La dinámica de la supervivencia difirió de forma marcada entre sitios (Figura 1a). En El Foyel (ladera norte), el porcentaje de plantines vivos se mantuvo relativamente constante a lo largo del experimento (Tabla 1) y el efecto del tiempo no fue significativo ( $\beta_{1}$ en Tabla A2 del Material Suplementario). En Los Repollos (fondo de valle) la tasa de supervivencia disminuyó drásticamente a lo largo del tiempo (Figura 1a), es decir, se observó interacción negativa entre tiempo y sitio $\left(\beta_{5}<0\right.$ en Tabla A2 del Material Suplementario). Si bien no encontramos efecto de la protección ( $\beta_{3}$ en Tabla A2 del Material Suplementario), el porcentaje observado de individuos vivos tendió a ser siempre más alto en los plantines protegidos (Tabla 1). En éstos, la supervivencia al final del experimento fue mayor al $70 \%$ en 
El Foyel y cercana al 15\% en Los Repollos, observándose porcentajes algo más bajos en los plantines sin protección (Tabla 1 ). La edad del plantín no afectó la supervivencia ( $\beta_{4}$ en Tabla A2 del Material Suplementario).

\section{Crecimiento en altura}

Los incrementos anuales en altura tendieron a aumentar con el tiempo y fueron sistemáticamente mayores en los plantines sin protección (Tabla 1). En efecto, el crecimiento fue modelado adecuadamente por una función exponencial (Figura 2). De acuerdo al modelo ajustado, la altura promedio de los plantines al momento de la plantación era de $\sim 23 \mathrm{~cm}\left(\alpha_{0}\right.$ en Tabla A2 del Material Suplementario). El ritmo de crecimiento fue similar entre plantines de 3 y 4 años de edad ( $\beta_{2}$ en Tabla A2 del Material Suplementario). Los que no tenían protección crecieron a una tasa del $17 \%\left(\hat{\beta}_{0}=0.17\right.$; Tabla A2 del Material Suplementario), más alta
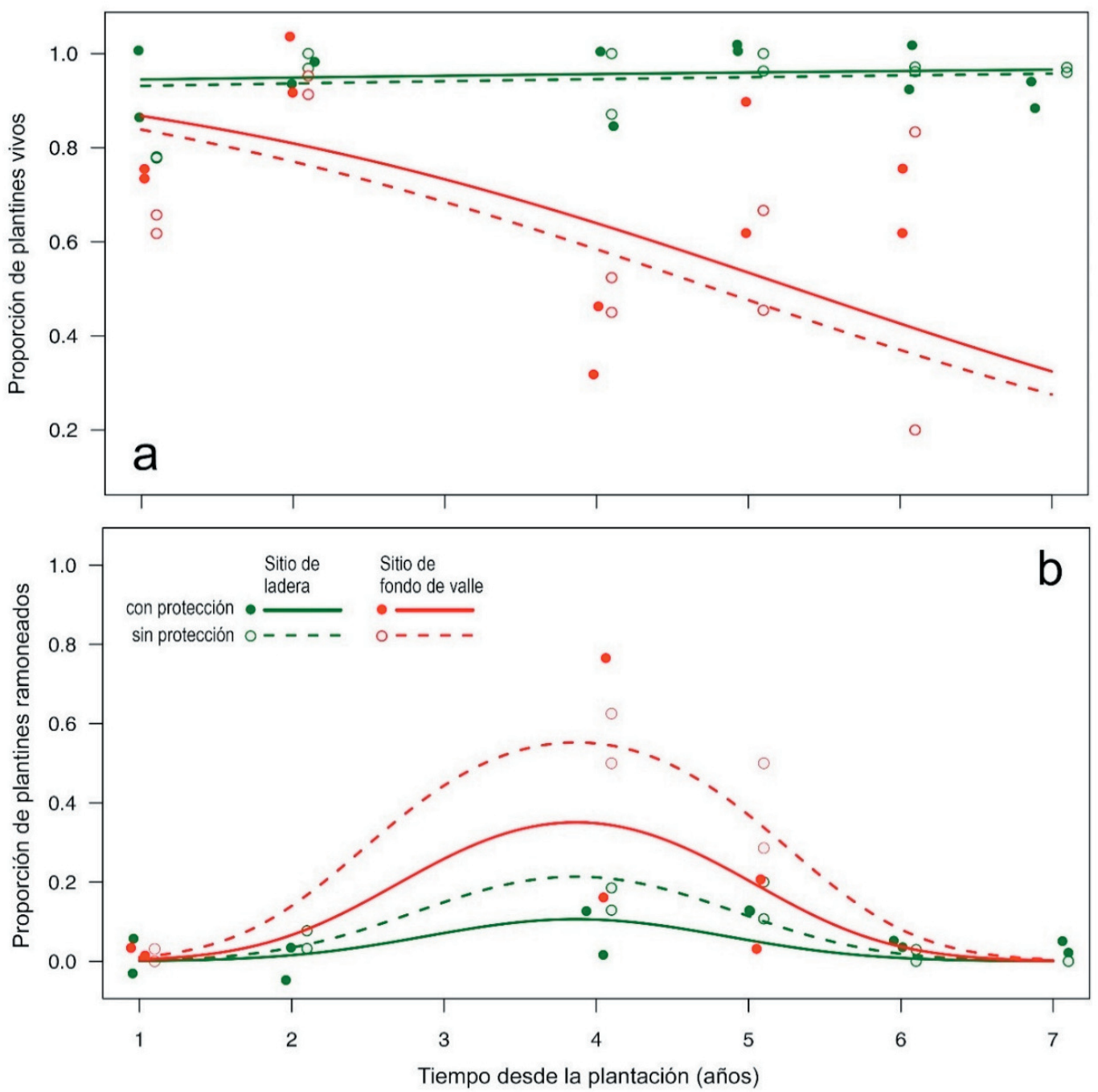

Figura 1. Dinámica de la supervivencia (a) y el ramoneo (b) de plantines de ciprés de la cordillera, con y sin protección contra herbívoros, en los dos sitios de plantación (El Foyel, un sitio de ladera, y Los Repollos, en un fondo de valle). Las curvas representan las probabilidades de supervivencia y ramoneo modeladas de acuerdo a modelos lineales generalizados (con estructura de errores cuasi-binomial en el primer caso y binomial en el segundo) y los puntos indican las proporciones observadas (de individuos vivos y plantines ramoneados, respectivamente). Los 8 puntos por año responden al diseño experimental: 2 sitios $\times 2$ tratamientos de protección $\times 2$ edades.

Figure 1. Survival (a) and browsing (b) dynamic of 'ciprés de la cordillera' seedlings, protected and non-protected from herbivores, in the two sites used for planting (El Foyel, a hillside site, and Los Repollos, in a valley bottom). Curves represent the survival and browsing probabilities as modeled from generalized linear models (with quasi-binomial error structure in the first case and binomial in the second one) and points indicate the observed proportions (live and browsed seedlings, respectively). The eight points per year reflect the experimental design: 2 sites $\mathrm{x} 2$ protection treatments $x 2$ ages. 
Tabla 1. Supervivencia y crecimiento (incremento) en altura observados en plantines con (c/p) y sin protección (s/p) en los dos sitios de plantación (El Foyel, sobre una ladera norte, y Los Repollos, en un fondo de valle) en relación con la precipitación y la temperatura anual registrada en el área de estudio (Sistema Nacional de Información Hídrica, Oficina Técnica Lago Puelo) durante el experimento.

Table 1. Survival and height growth (increment) observed in seedlings that were protected (c/p) and non-protected (s/p) from herbivores in the both plantation sites (El Foyel and Los Repollos, situated in a northern-exposure hillside and a valley bottom, respectively), related to precipitation and temperature in the study area (National Water Information System, Technical Office Lago Puelo) over the experiment.

\begin{tabular}{|c|c|c|c|c|c|c|c|c|}
\hline \multirow{3}{*}{ Año } & \multirow{3}{*}{$\begin{array}{c}\text { Precipitación } \\
\text { anual } \\
(\mathrm{mm})\end{array}$} & \multirow{3}{*}{$\begin{array}{c}\text { Temperatura } \\
\text { anual media } \\
\left({ }^{\circ} \mathrm{C}\right)\end{array}$} & \multicolumn{4}{|c|}{$\begin{array}{c}\% \text { Supervivencia } \\
\text { (vivos/plantados x 100) }\end{array}$} & \multirow{2}{*}{\multicolumn{2}{|c|}{$\begin{array}{c}\text { Crecimiento } \\
\text { (cm/año) } \\
\text { Ladera }\end{array}$}} \\
\hline & & & \multirow{2}{*}{\multicolumn{2}{|c|}{$\begin{array}{l}\text { Ladera } \\
\mathrm{s} / \mathrm{p}\end{array}$}} & \multicolumn{2}{|c|}{ Fondo de valle } & & \\
\hline & & & & & $\mathrm{s} / \mathrm{p}$ & $\mathrm{c} / \mathrm{p}$ & $\mathrm{s} / \mathrm{p}$ & $\mathrm{c} / \mathrm{p}$ \\
\hline 2009 & 936 & 11.0 & 78 & 92 & 64 & 73 & 4.1 & 3.1 \\
\hline 2010 & 641 & 10.8 & 77 & 90 & 59 & 73 & 6.3 & 5.5 \\
\hline 2012 & 690 & 12.2 & 72 & 82 & 29 & 27 & 6.1 & 5.1 \\
\hline 2013 & 840 & 12.4 & 70 & 82 & 10 & 20 & 12.2 & 7.8 \\
\hline 2014 & 810 & 11.4 & 69 & 79 & 9 & 14 & 9.3 & 7.0 \\
\hline 2015 & 941 & 12.4 & 66 & 72 & - & - & 10.5 & 7.6 \\
\hline \multicolumn{3}{|c|}{ Incremento anual medio (IMA) } & & & & & 8.1 & 6.0 \\
\hline
\end{tabular}

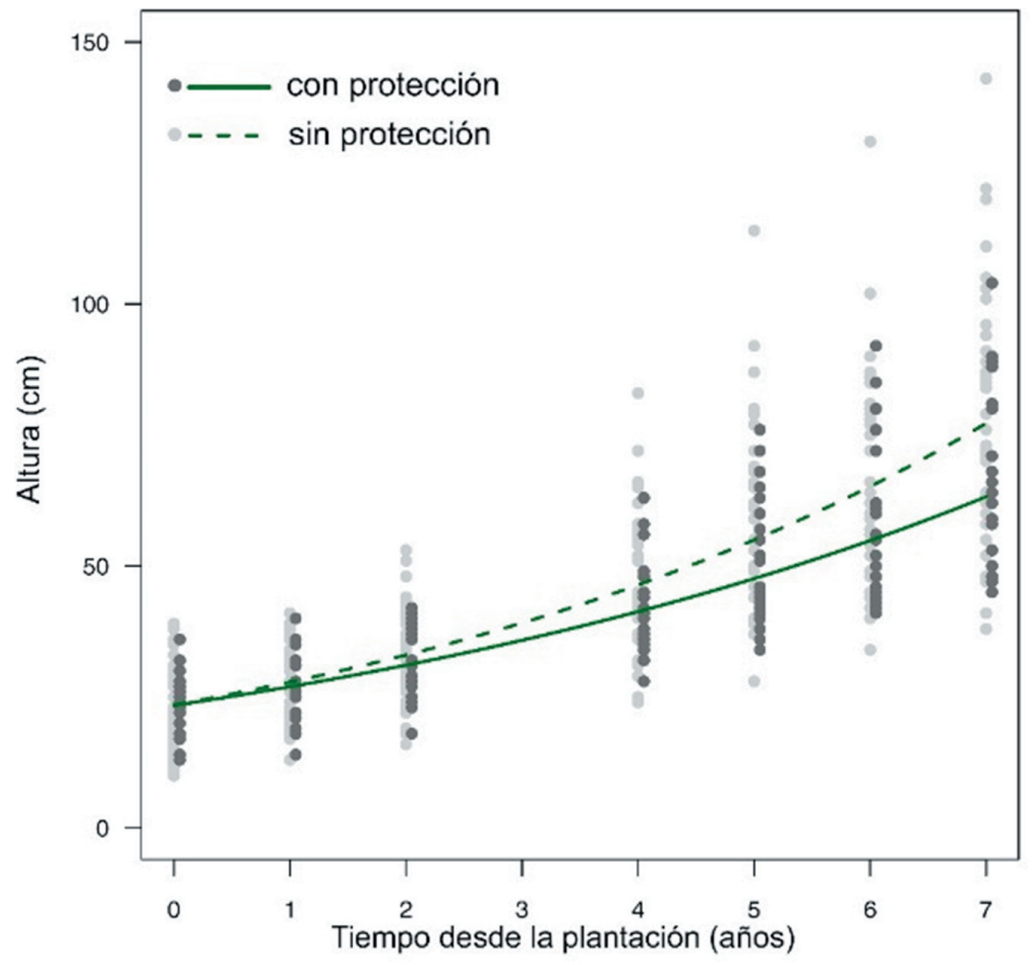

Figura 2. Crecimiento en altura de los plantines de ciprés de la cordillera en el sitio El Foyel, localizado sobre una ladera de exposición norte. En el panel superior se muestran las alturas observadas (puntos) y modeladas (curvas) por el modelo de crecimiento exponencial de efectos mixtos. El panel inferior muestra la distribución de las tasas de crecimiento de los plantines (efecto aleatorio) y el efecto (fijo) de la protección

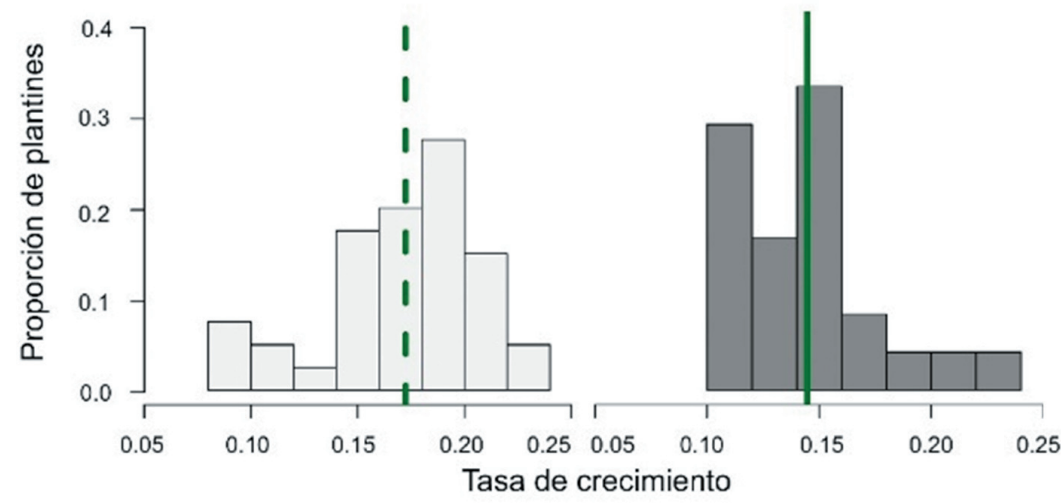
(líneas verticales).

Figure 2. Height growth of 'ciprés de la cordillera' seedlings in El Foyel, a northern-exposure hillside site. The upper panel shows the heights observed (points) and modeled with an exponential mixed-effects model (curves). The lower panel shows the distribution of growth rates of the seedlings (random effect) and the fixed effect of protection (vertical lines). 
que la de los plantines protegidos, es decir, la protección perjudicó el crecimiento $\left(\beta_{1}\right.$ en Tabla A2 del Material Suplementario). Estas tasas de crecimiento determinaron que al final del experimento (es decir, al séptimo año), los individuos protegidos alcanzaran una altura media de $\sim 65 \mathrm{~cm}$ y los no protegidos de $\sim 78$ cm (Figura 2).

\section{Ramoneo}

La tasa de ramoneo aumentó durante los primeros 4 años y luego comenzó a descender (Figura $1 b ; \beta_{1}>0$ y $\beta_{7}<0$ en Tabla A2 del Material Suplementario). Además, fue más alta en el fondo de valle que en la ladera y más baja en los plantines protegidos (Figura $1 \mathrm{~b} ; \beta_{2}>0$ y $\beta$ $>0$ en Tabla A2 del Material Suplementario). Al cuarto año, es decir, en el pico de la curva temporal, en Los Repollos, la tasa de ramoneo en los plantines sin protección fue de 0.55 y de 0.35 en los protegidos. En El Foyel, las tasas máximas fueron 0.21 y 0.11 , respectivamente. Al sexto año, la tasa de ramoneo descendió prácticamente a cero en todos los casos (Figura 1b). La edad del plantín no influyó en la probabilidad de ramoneo ( $\beta_{4}$ en Tabla A2 del Material Suplementario).

\section{Discusión}

El patrón temporal de la supervivencia mostró diferencias sustanciales entre sitios. En El Foyel, los plantines lograron establecerse con valores de supervivencia relativamente altos, lo cual era esperable, ya que las laderas asoleadas son beneficiosas para el desarrollo de la especie (Donoso Zegers 2013). En este sitio, la supervivencia al segundo año $(90 \%$ y $77 \%$ en plantines con y sin protección, respectivamente) (Tabla 1 ), tiempo en el que culminan en general este tipo de experimentos, se encontró dentro del rango observado en plantación bajo bosques de ciprés (ver Urretavizcaya et al. 2015 y referencias allí contenidas). A diferencia de estos trabajos, nuestroestudiosellevóadelanteen ecosistemas de matorral y provee la curva temporal hasta 7 años luego de la plantación, la cual en el sitio de ladera mostró una tendencia estable a lo largo del período de análisis (Figura 1a). En cambio, la alta mortalidad observada en Los Repollos pone en duda la factibilidad del ciprés para enriquecer (o producir en) este tipo ambientes. Estudios previos muestran que estos sitios tampoco son propicios para las plantaciones de algunas coníferas de rápido crecimiento tales como el pino Oregón (Pseudotsuga menziesii (Mirb.) Franco) (Davel
2008). Es interesante notar que si bien al segundo año los valores de supervivencia son relativamente altos (superior al $70 \%$ en plantines protegidos) (Tabla 1), sólo 10-15\% de los plantines permanecían vivos al culminar el monitoreo, lo cual resalta la importancia de los estudios temporales de mediano plazo basados en experimentos de campo.

En algunos sitios del norte de la Patagonia, la clausura individual de los plantines no mostró ser una técnica totalmente efectiva contra el ramoneo (Rusch et al. 2017). En este estudio, si bien no detectamos estadísticamente el efecto de la protección, la supervivencia fue sistemáticamente más alta en los plantines protegidos (Tabla 1). Al evaluar el patrón de herbivoría, observamos que la protección sí disminuyó la tasa de ramoneo, que aumentó con el tiempo desde la plantación hasta un máximo y luego comenzó a disminuir (Figura 1b). Durante los primeros años, el aumento del ramoneo puede responder al crecimiento del plantín; al adquirir mayor tamaño, las plantas se vuelven más aparentes para los animales ramoneadores como las liebres y, por ende, la probabilidad de ser detectadas y consumidas puede aumentar (Stutz et al. 2016). El momento de máxima tasa de ramoneo ocurrió al cuarto año, cuando la altura promedio fue aproximadamente 40 $\mathrm{cm}$ en los plantines protegidos y $45 \mathrm{~cm}$ en aquellos que no tuvieron protección. El ramoneo prácticamente cesó al sexto año, con una altura promedio entre los 50 y 60 $\mathrm{cm}$, rango en el que las plantas dejan de ser atacadas debido al tamaño corporal de la liebre (Bonino 2009). Ahora bien, que la protección haya reducido el ramoneo y que esto no se haya traducido en un aumento significativo de la supervivencia indicaría que el daño no es suficiente para determinar la mortalidad del plantín. No obstante, al presentar menos daño por ramoneo, los plantines protegidos podrían formar un fuste de mejor calidad lo que resultaría en un aumento del volumen maderable en plantaciones con objetivos de producción. De todas formas, esto ocurriría si los plantines son mayoritariamente ramoneados en las yemas apicales (i.e., sobre yemas laterales probablemente no afecte la forma del fuste), aspecto no considerado en nuestro estudio pero que sería interesante de evaluar en un futuro. Por otro lado, la tasa de ramoneo fue mayor en Los Repollos (Figura $1 b)$, el que menos diversidad de especies leñosas y potencial productivo presenta de ambos sitios de ensayo (Goldenberg et al. 2020c). La liebre europea modifica su 
dieta de acuerdo con la oferta de recursos forrajeros (Bonino et al. 1997). Ante la menor oferta de forraje de Los Repollos, los plantines probablemente se hayan presentado relativamente más atractivos para la liebre que en El Foyel, lo que explicaría la mayor tasa de ramoneo observada en el fondo de valle.

El ritmo medio de crecimiento en altura en el sitio de ladera fue comparable a aquellos de plantaciones bajo bosque de ciprés. Allí se han observado incrementos medios anuales (IMA) de entre 10 y $15 \mathrm{~cm} /$ año (Urretavizcaya et al. 2015), algo superiores a los registrados en El Foyel (Tabla 1). Lógicamente, el crecimiento observado en este estudio es mucho más lento al alcanzado por las coníferas exóticas plantadas en la región. En ensayos de 7 años con Pinus ponderosa en sitios con similar precipitación a la de Los Repollos se registraron IMAs de 22-30 cm/año (Andenmatten and Letourneau 2008), y en sitios de calidad media, las plantaciones de esta especie a los 10 años alcanzan alturas promedio de $3.5 \mathrm{~m}$, es decir, IMAs de 35 cm/año (Gonda 2001). Un aspecto interesante del modelo ajustado es que nos permitió cuantificar la tasa de crecimiento (proporción/porcentaje de incremento en altura, parámetro $b$ del modelo exponencial) de cada plantín (Figura 2$)$, su variabilidad $\left(\sigma_{b}\right.$ en Tabla A2 del Material Suplementario), y los posibles factores que la explican (protección y edad en nuestro caso). Las tasas de crecimiento rondaron el 15\% y disminuyeron con la protección. El efecto negativo de la protección también parece haber ocurrido en Los Repollos, en donde el crecimiento promedio al primer fue de $2.8 \mathrm{~cm}$ y $3.5 \mathrm{~cm}$ en los plantines protegidos y no protegidos, respectivamente (datos no mostrados). El tipo de protector usado (metálico) modifica las condiciones internas de luz y temperatura, y también puede afectar el desarrollo del plantín por daño físico (Sharew and Hairston-Strang 2005). Si el sombreo impuesto por la clausura es excesivo, el crecimiento puede verse afectado (Letorneau et al. 2019). Reducir la tasa de crecimiento en etapas tempranas es perjudicial no sólo en términos de incremento de existencias, sino que también prolonga el tiempo en que los plantines son susceptibles al daño por ramoneo.

\section{Conclusiones}

Este estudio describió, durante siete años, la dinámica de dos variables ecológicas de interés aplicado, la supervivencia y el crecimiento, en plantines de ciprés de la cordillera en dos sitios de matorral contrastantes de la Patagonia norte. En el sitio ubicado en el fondo de valle, donde la especie no se encuentra en forma natural, la mortalidad fue relativamente baja al principio, pero su incremento subsiguiente fue sustancial y sobrevivieron pocos individuos. Por el contrario, en la plantación sobre la ladera, donde la especie sí está presente en forma natural, la supervivencia se mantuvo estable. En este sitio, los valores de supervivencia y crecimiento estuvieron dentro de lo esperado si se los compara con plantaciones bajo bosque de ciprés, lo cual sugiere que la especie podría utilizarse para restaurar laderas de exposición norte como las del sitio de ensayo, brindando a su vez una alternativa de producción sustentable. La protección redujo el daño por ramoneo, que tuvo un máximo al cuarto año, cesó al sexto, y fue más alto en el sitio fondo de valle que en la ladera, pero atenuó la tasa de crecimiento. De esta forma, no es clara la conveniencia de proteger los plantines mediante la tecnología usada; futuros estudios deberían avanzar en el desarrollo de técnicas para asegurar el establecimiento y reducir el ramoneo sin afectar el crecimiento (una opción podría ser el uso protectores individuales sólo durante las primeras temporadas). El ciprés de la cordillera es uno de los árboles nativos de mayor valor maderero de la Patagonia norte $\mathrm{y}$, por lo tanto, estudiar la dinámica de los plantines es de interés regional para la conservación y el manejo forestal. De esta forma, si bien en este estudio sólo se ensayaron dos sitios que cubrieron una superficie de plantación reducida y los resultados no se deberían generalizar, lo encontrado podría contribuir con la gestión sustentable de los matorrales mixtos y los bosques de la región.

Agradecimientos. A Ayelén I. Carrón y Santiago A. Varela por la cesión de los registros edáficos y meteorológicos, respectivamente. Este trabajo fue financiado con fondos de los proyectos SILVA 020 (GEF TF 090118 - SILVA - MAGyP) y PIA 10058 (BIRF LN 7520 AR - CONICET).

\section{ReFERENCIAS}

Aparicio, A. G., M. J. Pastorino, and L. A. Gallo. 2009. Variación en el crecimiento temprano en altura de orígenes marginales esteparios de ciprés de la cordillera. Ciencia e Investigación Forestal 15:19-32.

Aparicio, A. G., M. J. Pastorino, and L. A. Gallo. 2010. Genetic variation of early height growth traits at the xeric limits of 
Austrocedrus chilensis (Cupressaceae). Austral Ecology 35:825-836. https://doi.org/10.1111/j.1442-9993.2009.02090.x.

Andenmatten, E., and F. J. Letourneau. 2008. Mejora del crecimiento inicial de pino ponderosa, por efecto del manejo de la vegetación en el sitio de plantación. Presencia 52:8-11.

Bonino, N. A. 2009. Liebres y conejos como plagas de plantaciones forestales. Serie técnica: Manejo Integrado de Plagas Forestales INTA EEA Bariloche. Cuadernillo $\mathrm{N}^{\circ} 7$.

Bonino, N. A., A. Sbriller, M. M. Manacorda, and F. Larosa. 1997. Food partitioning between the mara (Dolichotis patagonum) and the introduced hare (Lepus Europaeus) in the Monte desert, Argentina. Stud Neotrop Fauna and Environm 32:129-134. https://doi.org/10.1080/01650521.1997.9709614.

CIEFAP and MAyDS. 2016. Actualización de la Clasificación de Tipos Forestales y Cobertura del Suelo de la Región Bosque Andino Patagónico. Informe Final. Buenos Aires, Argentina.

Davel, M. 2008. Establecimiento y manejo del pino oregón en Patagonia. Manual Nº9. CIEFAP, Esquel.

De Paz, M., M. Gobbi, and E. Raffaele. 2019. Revisión de las experiencias de revegetación con fines de restauración en bosques de la Argentina. Ecología Austral 29:194-207. https://doi.org/10.25260/EA.19.29.2.0.689.

Donoso Zegers, C. 2013. Las especies arbóreas de los bosques templados de Chile y Argentina: autoecología. Marisa Cúneo Ediciones, Valdivia.

FAO. 2018. The State of the World's Forests 2018 - Forest pathways to sustainable development. Rome.

Fundación INVAP y CIEFAP. 2019. Compilación Integral de Datos, bajo metodología WISDOM, en la región de la Comarca Andina Ampliada, de oferta y demanda de biomasa relativa a cubrir necesidades energéticas, proyectando su evolución mediante modelos de crecimiento. Informe Final.

Gizachew, B., A. Brunner, and B. Øyen. 2012. Stand responses to initial spacing in Norway spruce plantations in Norway. Scandinavian Journal of Forest Research 27:637-648. https://doi.org/10.1080/02827581.2012.693191.

Goldenberg, M. G., Y. A. Cardoso, F. J. Oddi, and L. A. Garibaldi. 2020a. Fuelwood energy characteristics and biomass equations of the dominant species of northern Patagonia shrublands. Southern Forest 82:56-64. https://doi.org/10.2989/ 20702620.2019.1686693.

Goldenberg, M. G., F. J. Oddi, J. H. Gowda, and L. A. Garibaldi. 2020b. Effects of firewood harvesting intensity on biodiversity and ecosystem services in shrublands of northern Patagonia. Forest Ecosystems 7:47. https://doi.org/ 10.1186/s40663-020-00255-y.

Goldenberg, M. G., F. J. Oddi, M. M. Amoroso, and L. A. Garibaldi. 2020c. Effects of harvesting intensity and site conditions on biomass production of northern Patagonia shrublands. European Journal of Forest Research (en prensa). https://doi.org/10.1007/s10342-020-01292-6.

Goldenberg, M. G., J. H. Gowda, C. Casas, and L. A. Garibaldi. 2018. Efecto de la tasa de descuento sobre la priorización de alternativas de manejo del matorral Norpatagónico argentino. Bosque 39:217-226. https://doi.org/10.4067/S071792002018000200217.

Gonda, H. 2001. Manejo de pino ponderosa. Modelo preliminar para plantaciones en sitios de calidad media en la Patagonia andina. Patagonia Forestal 3:7-10.

Gowda, J., L. A. Garibladi, G. Pirk, M. Blackhall, E. Chaneton, M. de Paz, S. Díaz, G. Galende, N. Mazía, J. Paritsis, E. Raffaele, M. A. Relva, and Y. Sasal. 2014. Herbívoros: Actores Claves. Pp. 91-112 in E. Raffaele, M. de Torres Curth, C. L. Morales, and T. Kitzberger (eds.). Ecología e historia natural de la Patagonia Andina: un cuarto de siglo de investigación en biogeografía, ecología y conservación. Fundación de Historia Natural Félix de Azara, Ciudad Autónoma de Buenos Aires.

Goya, J., P. Yapura, J. Ferrando, M. Barrera, and M. Arturi. 1998. Crecimiento individual y de rodales de Austrocedrus chilensis en Bolsón, Río Negro, Argentina. Revista de la Facultad de Agronomía Universidad Nacional de La Plata (Argentina) 103:149-155.

Grosfeld, J., L. Chauchard, and J. H. Gowda. 2019. ¿Podemos manejar sustentablemente el bosque nativo de Patagonia Norte? Ecología Austral 29:156-163. https://doi.org/10.25260/EA.19.29.1.0.775.

Gyenge, J. E., M. E. Fernández, M. Sarasola, M. de Urquiza, and T. Schlichter. 2009. Ecuaciones para la estimación de biomasa aérea y volumen de fuste de algunas especies leñosas nativas en el valle del río Foyel, NO de la Patagonia argentina. Bosque 30:95-101. https://doi.org/10.4067/S0717-92002009000200005.

Kitzberger, T., D. Steinaker, and T. T. Veblen. 2000. Effects of climatic variability on facilitation of tree establishment in northern Patagonia. Ecology 81:1914-1924. https://doi.org/10.1890/0012-9658(2000)081[1914:EOCVOF]2.0.CO;2.

Kitzberger, T., and T. T. Veblen. 1999. Fire-induced changes in northern Patagonian landscapes. Landscape Ecology 14:1-15. https://doi.org/10.1023/A:1008069712826.

Montagnini, F., D. Cusack, B. Petit, and M. Kanninen. 2004. Environmental Services of Native Tree Plantations and Agroforestry Systems in Central America. Journal of Sustainable Forestry 21:51-67. https://doi.org/10.1300/ J091v21n01_03.

Navarrete Espinoza, E., J. Cárcamo Ojeda, and P. Novoa Barra. Modelos de crecimiento diametral para Austrocedrus chilensis en la Cordillera de Nahuelbuta, Chile: Una interpretación biológica. Ciencia e Investigación Agraria 33: 311-320.

Núñez, C., E. Raffaele, M. A. Núñez, and F. Cuassolo. 2009. When do nurse plants stop nursing? Temporal changes in water stress levels in Austrocedrus chilensis growing within and outside shrubs. Journal of Vegetation Science 20: 1064-1071. https://doi.org/10.1111/j.1654-1103.2009.01107.x.

Landesmann, J. B., J. H. Gowda, and T. Kitzberger. 2016. Temporal shifts in the interaction between woody resprouters and an obligate seeder tree during a post-fire succession in Patagonia. Journal of Vegetation Science 27:1198-1208. 
https://doi.org/10.1111/jvs.12430.

Letourneau, F. J., G. Namiot, M. H. Perdomo, and J. G. Basil. 2019. Efecto de la aclimatación a la intensidad lumínica en vivero sobre el desempeño de plantines de Austrocedrus chilensis en plantación. Revista de Investigaciones Agropecuarias 45:216-224.

Oddi, F. J., F. Miguez, L. Ghermandi, L. O. Bianchi, and L. A. Garibaldi. 2019. A nonlinear mixed-effects modelling approach for ecological data: Using temporal dynamics of vegetation moisture as an example. Ecology and Evolution 9:10225-10240. https://doi.org/10.1002/ece3.5543.

Oliver, C. D., and B. C. Larson. 1996. Forest Stand Dynamics (updated edition). John Wiley, New York.

Pastorino, M. J., P. Marchelli, M. Milleron, C. Soliani, and L. A. Gallo. 2009. The effect of different glaciation patterns over the current genetic structure of the southern beech Nothofagus Antarctica. Genetica 136:79-88. https://doi.org/ 10.1007/s10709-008-9314-2.

Pafundi, L., M. F. Urretavizcaya, and G. E. Defosse. 2014. Improving survival and growth of planted Austrocedrus chilensis seedlings in disturbed Patagonian forests of Argentina by managing understory vegetation. Environmental Management 54:1412-1420. https://doi.org/10.1007/s00267-014-0363-2.

Pérez-Ramos, I., and T. Marañón. 2001. Community-level seedling dynamics in Mediterranean forests: uncoupling between the canopy and the seedling layers. Journal of Vegetation Science 23:526-540. https://doi.org/10.1111/j.16541103.2011.01365.x.

Peri, P. L., G. J. Martínez Pastur, L. Monelos, and M. Beroiz. 2013. La distribución más oriental de Nothofagus antarctica en el Río Gallegos (Santa Cruz). Anales del Instituto de la Patagonia 41:113-117. https://doi.org/10.4067/S0718686X2013000100010.

Pinheiro, J. C., and D. M. Bates. 2000. Mixed-effects models in S and SPLUS. New York: Springer-Verlag. https://doi.org/ 10.1007/978-1-4419-0318-1.

Pinheiro, J. C., D. M. Bates, S. DebRoy, D. Sarkar, and R Core Team. 2016._nlme: Linear and Nonlinear Mixed Effects Models_. R package version 3.1-126. URL: CRAN.R-project.org/package=nlme.

Promis, Á. Plantaciones nativas o exóticas: Reflexiones sobre los impactos ambientales en Chile. Ecología Austral 30: 191-198. https://doi.org/10.25260/EA.20.30.2.0.1064.

Puettmann, K. W. 20011. Silvicultural Challenges and Options in the Context of Global Change: "Simple" Fixes and Opportunities for New Management Approaches. Journal of Forestry 109:321-331.

R Core Team. 2018. R: A language and environment for Statistical Computing. R Foundation for Statistical Computing. Vienna, Austria. URL: www.R-project.org.

Ramírez, C., M. Correa, H. Figueroa, and J. San Martín. 1985. Variación del hábito y hábitat de Nothofagus antarctica en el sur de Chile. Bosque 6:55-73. https://doi.org/10.4206/bosque.1985.v6n2-01.

Relva, M. A., and L. A. Sancholuz. 2000. Effects of simulated browsing on the growth of Austrocedrus chilensis saplings. Plant Ecology 151:121-127. https://doi.org/10.1023/A:1026505412732.

Relva, M. A., and T. T. Veblen. 1998. Impacts of large herbivores on A. chilensis forests in Patagonia, Argentina. Forest Ecology and Management 108:27-40. https://doi.org/10.1016/S0378-1127(97)00313-7.

Reque, J. A, M. Sarasola, and M. E. Fernández. 2007. Caracterización silvícola de ñirantales del norte de la Patagonia para la gestión forestal sostenible silvicultural. Bosque 28:33-45. https://doi.org/10.4067/S0717-92002007000100006.

Rovere, A., M. E. Gobbi, and M. A. Relva. 2004. Regeneración de Austrocedrus chilensis. Pp. 1-16 in M. Arturi, J. Frangi and J. Goya (eds.). Ecología y Manejo de Bosques de Argentina. Editorial de la Universidad Nacional de La Plata (EDULP), La Plata.

Rusch, V., D. R. López, L. Cavallero, G. M. Rusch, L. A. Garibaldi, and P. Peri. 2017. Modelo de estados y transiciones de los ñirantales del NO de la Patagonia como herramienta para el uso silvopastoril sustentable. Ecología Austral 27:266-278. https://doi.org/10.25260/EA.17.27.2.0.240.

Sharew, H., and A. Hairston-Strang. 2005. A Comparison of Seedling Growth and Light Transmission among Tree Shelters. Northern Journal of Applied Forestry 22:102-110. https://doi.org/10.1093/njaf/22.2.102.

Steffan-dewenter, I., M. Kessler, J. Barkmann, M. M. Bos, D. Buchori, S. Erasmi, H. Faust, G. Gerold, K. Glenk, S. R. Gradstein, E. Guhardja, M. Harteveld, D. Hertel, P. Höhn, M. Kappas, S. Köhler, C. Leuschner, M. Maertens, R. Marggraf, S. Migge-Kleian, J. Mogea, R. Pitopang, M. Schaefer, S. Schwarze, S. G. Sporn, A. Steingrebe, S. S. Tjitrosoedirdjo, S. Tjitrosoemito, A. Twele, R. Weber, L. Woltmann, M. Zeller, and T. Tscharntke. 2007. Tradeoffs between income, biodiversity, and ecosystem functioning during tropical rainforest conversion and agroforestry intensification. Proceedings of the National Academy of Sciences of the USA 104:4973-4978. https://doi.org/10.1073/ pnas.0608409104.

Stutz, R. S., B. M. Croak, N. Proschogo, P. B. Banks, and C. McArthur. 2016. Olfactory and visual plant cues as drivers of selective herbivory. Oikos 126:2. https://doi.org/10.1111/oik.03422.

Urretavizcaya, M. F., M. Pastorino, V. Mondino, and L. Contardi. 2015. La plantación con árboles nativos. Pp. 335-368 in L. Chauchard, M. C. Furgoni, and C. Nowak (eds.). Manual de Buenas Prácticas para el manejo de plantaciones forestales en el noroeste de la Patagonia. Buenos Aires.

Veblen, T. T., B. R. Burns, T. Kitzberger, A. Lara, and R. Villalba. 1995. The Ecology of the conifers of Southern South America. Pp. 120-155 in N. J. Enright, and R. S. Hills (eds). Ecology of the southern conifers. Melbourne University Press, Carlton.

Veblen, T., C. Donoso, T. Kitzberger, and A. Rebertus. 1996. Ecology of southern Chilean and Argentinean Nothofagus Forests. Pp. 293-353 in T. Veblen, R. S. Hill, and J. Read (eds). The ecology and biogeography of Nothofagus forests. 
Yale University Press, New Haven, USA.

Williams-Linera, G., C. Álvarez-Aquino, A. Suárez, C. Blundo, C. Smith-Ramírez, C. Echeverria, E. Cruz-Cruz, G. Bolados, J. J. Armesto, K. Heinemann, L. Malizia, P. Becerra, R. F. del Castillo, and R. Urrutia. 2011. Experimental Analysis of Dryland Forest Restoration Techniques. Pp. 131-181 in A. C. Newton, and N. Tejedor (eds.). Principles and Practice of Forest Landscape Restoration: Case studies from the drylands of Latin America. IUCN, Gland, Switzerland.

Zeide, B. 2004. Optimal stand density: a solution. Canadian Journal of Forest Research 34:846-854. https://doi.org/ $10.1139 / x 03-258$. 